\title{
Detection of epistasis and linkage of interacting genes in the presence of reciprocal differences
}

\author{
H. S. Pooni, D. T. Coombs, ${ }^{*}$ \\ P. S. Virk and J. L. Jinks
}

Department of Genetics, University of Birmingham, P.O. Box 363, Birmingham B15 2TT, U.K.

Scaling tests of Mather and Jinks $(1971,1982)$ and modified tests of Jinks (1978) have been applied to the mean performance and environmental sensitivity measurements of the $P_{1}, P_{2}, F_{1}\left(P_{1} \times P_{2}\right), R F_{1}\left(P_{2} \times P_{1}\right), F_{2}\left(F_{1} \times F_{1}\right), B_{1}\left(P_{1} \times\right.$ $\left.F_{1}\right), R B_{1}\left(F_{1} \times P_{1}\right), B_{2}\left(P_{2} \times F_{1}\right), R B_{2}\left(F_{1} \times P_{2}\right), F_{3}\left(F_{2}\right.$ self $), L_{1}\left(F_{2} \times P_{1}\right), L_{2}\left(F_{2} \times P_{2}\right)$ and $L_{3}\left(F_{2} \times F_{1}\right)$ generations derived from a cross between a flue cured variety SCR and burley variety $S 3$ of Nicotiana tabacum to detect the presence of non-allelic interactions, genotype $\times$ environmental interactions, reciprocal effects and linkage of the interacting genes prior to the estimation of the components of first degree statistics by the method of weighted least squares. Fifteen characters representing growth rate, maturity, yield and yield components have been analysed. In each case the tests proved useful in identifying the presence or absence of epistasis, linkage of interacting genes and reciprocal effects thus illustrating once more that simple statistical tests are in general more sensitive and more specific than sequential model fitting procedures for detecting these complex effects. This investigation also revealed that epistasis, linkage of interacting genes, reciprocal differences and their interactions contribute more frequently to the variability in mean performance than in environmental sensitivity.

\section{INTRODUCTION}

Mather's (1949) scaling tests for detecting epistasis and Jinks' (1978) unambiguous tests for detecting linkage of the genes showing digenic interactions have been available for sometime. They allow one to determine which genetical components are contributing to the genetical variability among first degree statistics such as generation means at a significant level. Thus, they help to identify the components of an adequate genetical model prior to obtaining weighted least squares estimates. However, both tests assume that differences between reciprocal crosses have already been shown to be non-significant using the appropriate scaling tests (Mather and Jinks, 1971, 1982). In their original form the tests for epistasis are therefore invalidated whenever there are significant differences between reciprocal crosses. Alternative scaling tests are, nevertheless, available which can be used in these circumstances (see Mather and Jinks 1971, 1982). The same procedures can be extrapolated to the detection of linkage between

\footnotetext{
* Present address: Nickerson RBP Ltd., Joseph Nickerson Research Centre, Rothwell, Lincoln LN7 6DT.
}

epistatic genes in the presence of reciprocal differences. In this paper, we therefore propose alternatives to the $\bar{B}_{1}-\bar{L}_{1}, \bar{B}_{2}-\bar{L}_{2}$ and $\bar{F}_{2}-\bar{L}_{3}$ comparisons of Jinks (1978) and apply them to experimental data for the first time. We also apply the alternative scaling tests of Mather and Jinks $(1971,1982)$ and two additional comparisons that are suitable to test for non-allelic interactions in the presence of reciprocal differences. These and other analyses will be carried out to interpret the genetical control of variability in a cross of two inbred varieties of Nicotiana tabacum.

\section{MATERIALS AND METHODS}

The materials are derived from a cross between two commercial varieties of Nicotiana tabacum, namely SCR, a German flue-cured variety which was bred from an old U.S.A. variety known as "Golden Harvest", and S3, and air-cured burley variety of Swiss origin. Both varieties have been maintained by controlled selfing for at least six generations and therefore can be classified as pure breeding lines. Seeds of these varieties were provided by Carrearas Rothmans Limited in 1976 
(Coombs, 1980). Individual plants of the parental varieties (SCR and S3) and their $F_{1}(\mathrm{SCR} \times \mathrm{S} 3)$ were selfed and intercrossed during the summer of 1977 to produce the seeds of the parental, $F_{1}(\mathrm{SCR} \times \mathrm{S} 3), \quad R F_{1}(\mathrm{~S} 3 \times \mathrm{SCR}), \quad F_{2}\left(F_{1}\right.$ selfed $)$ $B_{1}\left(\mathrm{SCR} \times F_{1}\right), \quad R B_{1}\left(F_{1} \times \mathrm{SCR}\right), B_{2}\left(\mathrm{~S} 3 \times F_{1}\right)$ and $R B_{2}\left(F_{1} \times \mathrm{S} 3\right)$ generations. Twenty-three randomly chosen $F_{2}$ plants were also selfed and individually crossed to $\mathrm{SCR}, \mathrm{S} 3$ and $F_{1}(\mathrm{SCR} \times \mathrm{S} 3)$ to produce the $F_{3}$ families and the $L_{1 i}, L_{2 i}$ and $L_{3 i}$ families of a triple test cross (Kearsey and Jinks, 1968). At least two flowers were used for each selfing or cross pollination to minimise the capsule differences, if any. These materials were grown in duplicate blocks each containing 2480 individually randomised plants. Randomisation was carried out at the time of sowing to avoid any sharing of common environment in the early stages of development. The details of the distribution of these plants amongst various families and families within generations in each block are given in table 1 . Block 1 was raised in the University field at normal experimental density $(106 \mathrm{~cm}$ between rows and $30 \mathrm{~cm}$ between plants within rows) and block 2 was grown at Avoncroft at commercial growing density $(106 \mathrm{~cm}$ between rows and $15 \mathrm{~cm}$ between plants within rows). The experiment was conducted during the summer of 1978 and blocks were sown on consecutive days.

Fifteen metrical traits listed in table 2 and one qualitative character (burley vs. non-burley) were scored on all plants individually. Eleven of these traits were scored in the field and the rest were derivatives. The data were processed through the Birmingham University Multics Computer.

Table 1 Dimensions of the experiment per density

\begin{tabular}{lccc}
\hline Generation & $\begin{array}{l}\text { Number of } \\
\text { families }\end{array}$ & $\begin{array}{l}\text { Family } \\
\text { size }\end{array}$ & $\begin{array}{l}\text { Total } \\
\text { number of } \\
\text { plants }\end{array}$ \\
\hline SCR & 1 & 40 & 40 \\
S3 & 1 & 40 & 40 \\
$F_{1}(\mathrm{SCR} \times \mathrm{S} 3)$ & 1 & 40 & 40 \\
$R F_{1}(\mathrm{~S} 3 \times \mathrm{SCR})$ & 1 & 40 & 40 \\
$F_{2}\left(F_{1}\right.$ selfed $)$ & 1 & 160 & 160 \\
$B_{1}\left(\mathrm{SCR} \times F_{1}\right)$ & 1 & 80 & 80 \\
$R B_{1}\left(F_{1} \times \mathrm{SCR}\right)$ & 1 & 80 & 80 \\
$B_{2}\left(\mathrm{~S} 3 \times F_{1}\right)$ & 1 & 80 & 80 \\
$R B_{2}\left(F_{1} \times \mathrm{S} 3\right)$ & 1 & 80 & 80 \\
$F_{3}\left(F_{2}\right.$ selfed $)$ & 23 & 20 & 460 \\
$L_{1}\left(F_{2} \times \mathrm{SCR}\right)$ & 23 & 20 & 460 \\
$L_{2}\left(F_{2} \times \mathrm{S} 3\right)$ & 23 & 20 & 460 \\
$L_{3}\left(F_{2} \times F_{1}\right)$ & 23 & 20 & 460 \\
Total & & & 2480 \\
\hline
\end{tabular}

Table 2 A list of characters scored on the present material

\begin{tabular}{ll}
\hline Character & Description \\
\hline$H_{1}$ & Plant height $(\mathrm{cm})$ at 12 weeks after sowing \\
$H_{2}$ & Plant height $(\mathrm{cm})$ at 14 weeks after sowing \\
$L L$ & Leaf length $(\mathrm{cm})$ of the largest leaf blade at 15 \\
& weeks after sowing \\
$L B$ & Leaf breadth (cm) of the broadest leaf at 15 weeks \\
& after sowing \\
$H F T$ & Height in cm at the time of flowering \\
$F T$ & Flowering time in days from the date of sowing \\
$F H$ & Height of the plant at the end of the season $(\mathrm{cm})$ \\
$T Y$ & Total yield (g) \\
$S Y$ & Stem yield (g) \\
$L N$ & Number of leaves per plant counted from base \\
& to the flowering head \\
$L Y$ & Leaf yield (TY-SY) \\
$I N L$ & Internode length $(\mathrm{cm})$ calculated as: $F H / L N$ \\
$L R$ & Leaf ratio $=L L / L B$ \\
$L S R$ & Leaf: Stalk ratio $=L Y / S Y$ \\
$H A F$ & Height (cm) after flowering $(F H-H F T)$ \\
\hline
\end{tabular}

All analyses have been conducted on the mean performance and environmental sensitivity separately (Brumpton, Boughey and Jinks, 1977; Jinks, Jayasekara and Boughey, 1977). The measures of mean performance and environmental sensitivity of each generation were obtained from the sum and difference of its performance in the two densities as:

$$
\text { Mean performance }=\frac{1}{2}\left\{\bar{F}_{n}(N D)+\bar{F}_{n}(C D)\right\}
$$

and

Enrivonmental sensitivity $=\frac{1}{2}\left\{\bar{F}_{n}(N D)-\bar{F}_{n}(C D)\right\}$

where $\bar{F}_{n}(N D)$ and $\bar{F}_{n}(C D)$ are the means of the $n$th generation in the normal experimental and commercial growing densities, respectively.

\section{RESULTS}

\section{Reciprocal differences}

Initially, we compare the reciprocals of each generation to test for the presence of reciprocal differences following Mather and Jinks (1971, 1982). The values of $\bar{F}_{1}-R \bar{F}_{1}, \bar{B}_{1}-R \bar{B}_{1}$ and $\bar{B}_{2}-$ $R \bar{B}_{2}$, their standard errors and their significances are tabulated in table 3 for each combination of character/mean performance and character/environmental sensitivity. At least one of these comparisons takes a significant value for every character/mean performance combination. These differences can be attributed to a number of sources. The most important and contrasting are cytoplasmic effects and the delayed effects of 
Table 3 Tests of reciprocal differences in the $F_{1}$ and backcross generations

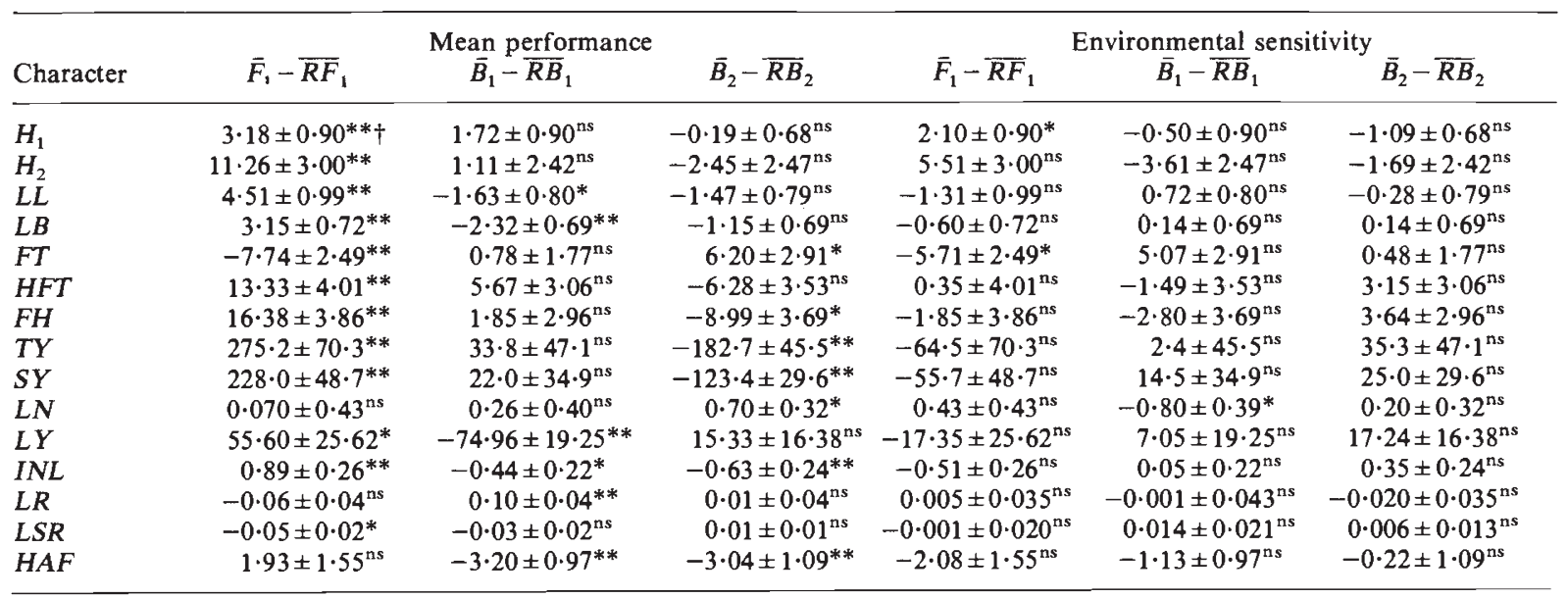

$\dagger$ ns $P>0.05 ; * 0.05 \geqq P>0.01 ; * * P \leqq 0.01$.

the maternal (or paternal) genotype. On a simple model in which the cytoplasmic effects are assumed to be transmitted unchanged by the maternal parent for an indeterminate number of generations the $\bar{F}_{1}-R \bar{F}_{1}$ and $R \bar{B}_{2}-\bar{B}_{2}$ comparisons are expected to be statistically significant and magnitudinally equal. Further the $\bar{B}_{1}-R \bar{B}_{1}$ comparison should always be non-significant. Reference to table 3 shows that this model fails to explain the reciprocal differences in nine characters.

On a simple additive dominance model based on the delayed effects of the maternal (or paternal) genotype on its progeny phenotype the $\bar{F}_{1}-R \bar{F}_{1}$, $\bar{B}_{1}-R \bar{B}_{1}$ and $\bar{B}_{2}-R \bar{B}_{2}$ comparisons provide tests of $2[d]_{m},[d]_{m}-[h]_{m}$ and $-[d]_{m}-[h]_{m}$, respectively (see Barnes, 1968 and Mather and Jinks, 1971 for definitions of $[d]_{m}$ and $[h]_{m}$ etc.). The results in table 3 confirm that $[d]_{m}$ is positive for ten characters and negative for flowering time $(F T)$ and leaf stem ratio $(L S R)$. Further, $[h]_{m}$ should be significant, positive and larger than $[d]_{m}$ for $L L, L B, L Y, I N L$ and $H A F$ because $\bar{B}_{1}-R \bar{B}_{1}$ takes a negative sign for each of these characters. However, this observation is not unconditionally supported by the $\bar{B}_{2}-R \bar{B}_{2}$ comparison which is non-significant for $L L, L B$ and $L Y$. Overall, $[d]_{m}$ appears to be significant more frequently than $[h]_{m}$.

For environmental sensitivity only three of the comparisons are significant, $\vec{F}_{1}-R \bar{F}_{1}$ for $H_{1}$ and $F T$ and $\bar{B}_{1}-R \bar{B}_{1}$ for $L N$. This suggests that the reciprocal differences do not respond differentially to the site and density effects for at least 12 of the 15 characters scored.

\section{Non-allelic interactions}

The absence of reciprocal differences for all but three of the combinations of character/environmental sensitivity means that the standard A, B, C and D scaling tests of Mather (1949) can be applied for detecting the presence of non-allelic interactions for 12 of these combinations. However, we have extended the definitions of the $A, B$ and $C$ scaling tests as follows to include the triple test cross families because $\bar{L}_{1}=\bar{B}_{1}, \bar{L}_{2}=\bar{B}_{2}$ and $\bar{L}_{3}=\bar{F}_{2}$ in the absence of epistasis and reciprocal differences.

Scaling test Comparison

$$
\begin{array}{ll}
\mathrm{A}^{\prime} & \bar{B}_{1}+\bar{L}_{1}-\bar{F}_{1}-\bar{P}_{1}=0 \\
\mathrm{~B}^{\prime} & \bar{B}_{2}+\bar{L}_{2}-\bar{F}_{1}-\bar{P}_{2}=0 \\
\mathrm{C}^{\prime} & 2 \bar{F}_{2}+2 \bar{L}_{3}-2 \bar{F}_{1}-\bar{P}_{1}-\bar{P}_{2}=0
\end{array}
$$

In each case these and Mather's (1949) scaling tests were applied to the means of reciprocal crosses. The results are presented in table 4 .

The presence of significant reciprocal differences for each of the character/mean performance and three of the character/environmental sensitivity combinations, however, invalidates the unambiguity of the $A, B$ and $C$ scaling tests even when they are applied to the overall means of the reciprocal crosses (see Mather and Jinks, 1971, 1982). Further, the relationships $\bar{L}_{1}=\bar{B}_{1}, \bar{L}_{2}=\bar{B}_{2}$ and $\bar{L}_{3}=\bar{F}_{2}$ are not expected to hold when reciprocal differences are significant. Therefore, following Mather and Jinks $(1971,1982)$ these tests have been applied in their modified form as 
Table 4 Scaling tests and their significance for all combinations of character/environmental sensitivity for which reciprocal differences are non-significant

\begin{tabular}{|c|c|c|c|c|c|c|c|}
\hline \multicolumn{8}{|c|}{ Scaling tests } \\
\hline Character & A & $\mathrm{A}^{\prime} \dagger$ & B & $\mathbf{B}^{\prime}$ & $\mathrm{C}$ & $\mathrm{C}^{\prime}$ & D \\
\hline $\mathrm{H}_{2}$ & $-4 \cdot 46 \pm 3 \cdot 19^{n s}$ & $-1 \cdot 63 \pm 2 \cdot 64^{\mathrm{ns}}$ & $-7 \cdot 78 \pm 3 \cdot 25^{*}$ & $-7 \cdot 65 \pm 2 \cdot 72^{* *}$ & $-10.93 \pm 6 \cdot 18^{\mathrm{ns}}$ & $-10.01 \pm 5.07^{*}$ & $-2 \cdot 04 \pm 7 \cdot 67^{\mathrm{ns}}$ \\
\hline$L B$ & $-0.51 \pm 0.95^{\mathrm{ns}}$ & $-0.11 \pm 0.78^{\mathrm{ns}}$ & $-0.27 \pm 0.89^{n s}$ & $0 \cdot 29 \pm 0.70^{\mathrm{ns}}$ & $-2 \cdot 47 \pm 1 \cdot 46^{\mathrm{ns}}$ & $-0.95 \pm 1 \cdot 21^{\mathrm{ns}}$ & $1 \cdot 41 \pm 1 \cdot 38^{\mathrm{ns}}$ \\
\hline HFT & $-5 \cdot 62 \pm 5 \cdot 91^{\mathrm{ns}}$ & $-7 \cdot 30 \pm 5 \cdot 32^{\mathrm{ns}}$ & $0 \cdot 74 \pm 4 \cdot 30^{\mathrm{ns}}$ & $3.04 \pm 3.94^{\mathrm{ns}}$ & $-6 \cdot 32 \pm 8 \cdot 89^{n s}$ & $-5 \cdot 06 \pm 7 \cdot 88^{\mathrm{ns}}$ & $3 \cdot 21 \pm 11 \cdot 99^{\mathrm{ns}}$ \\
\hline$F H$ & $0 \cdot 69 \pm 6 \cdot 21^{\mathrm{ns}}$ & $-3 \cdot 21 \pm 5 \cdot 56^{\mathrm{ns}}$ & $6 \cdot 23 \pm 4 \cdot 23^{\mathrm{ns}}$ & $8 \cdot 05 \pm 3 \cdot 88^{*}$ & $-2 \cdot 60 \pm 8 \cdot 90^{\text {ns }}$ & $0 \cdot 86 \pm 7 \cdot 73^{\mathrm{ns}}$ & $1 \cdot 89 \pm 11 \cdot 34^{\mathrm{ns}}$ \\
\hline$L Y$ & $-25 \cdot 10 \pm 30 \cdot 32^{\mathrm{ns}}$ & $-7 \cdot 94 \pm 26 \cdot 85^{\mathrm{ns}}$ & $0.97 \pm 24 \cdot 37^{\mathrm{ns}}$ & $7 \cdot 73 \pm 21 \cdot 48^{\mathrm{ns}}$ & $-3 \cdot 02 \pm 48 \cdot 67^{\mathrm{ns}}$ & $5 \cdot 84 \pm 40 \cdot 81^{\mathrm{ns}}$ & $-41 \cdot 61 \pm 48 \cdot 15^{\mathrm{ns}}$ \\
\hline$I N L$ & $0.67 \pm 0.29^{*}$ & $0.53 \pm 0.24^{*}$ & $0 \cdot 48 \pm 0 \cdot 38^{\mathrm{ns}}$ & $0 \cdot 22 \pm 0.34^{\mathrm{ns}}$ & $0.77 \pm 0.67^{\mathrm{ns}}$ & $0.96 \pm 0.53^{\text {ns }}$ & $0.31 \pm 0.76^{\mathrm{ns}}$ \\
\hline$L R$ & $-0.06 \pm 0.05^{\mathrm{ns}}$ & $-0.04 \pm 0.04^{\mathrm{ns}}$ & $-0.03 \pm 0.05^{\mathrm{ns}}$ & $-0.01 \pm 0.04^{\mathrm{ns}}$ & $-0.21 \pm 0.09^{*}$ & $-0.14 \pm 0.07^{*}$ & $0.04 \pm 0 \cdot 10^{\mathrm{ns}}$ \\
\hline$L S R$ & $-0 \cdot 06 \pm 0.04^{\mathrm{ns}}$ & $-0.04 \pm 0.03^{\mathrm{ns}}$ & $-0.03 \pm 0.02^{\mathrm{ns}}$ & $-0.02 \pm 0.02^{\mathrm{ns}}$ & $-0.09 \pm 0.05^{\mathrm{ns}}$ & $-0.07 \pm 0.04^{\mathrm{ns}}$ & $-0.06 \pm 0.05^{\mathrm{ns}}$ \\
\hline$H A F$ & $2 \cdot 34 \pm 1 \cdot 82^{\mathrm{ns}}$ & $2 \cdot 44 \pm 1 \cdot 68^{\mathrm{ns}}$ & $4 \cdot 45 \pm 1 \cdot 51^{* *}$ & $3 \cdot 37 \pm 1 \cdot 24^{* *}$ & $-0.44 \pm 3 \cdot 45^{\mathrm{ns}}$ & $2 \cdot 30 \pm 2 \cdot 69^{\mathrm{ns}}$ & $2 \cdot 22 \pm 2 \cdot 96^{\mathrm{ns}}$ \\
\hline
\end{tabular}

See table 3 for probability.

t See text for definitions of $A^{\prime}, B^{\prime}$ and $C^{\prime}$.

follows:

$$
\begin{array}{cc}
\begin{array}{c}
\text { Scaling } \\
\text { test }
\end{array} & \text { Comparison } \\
\mathrm{A}^{\prime \prime} & 2 \bar{B}_{1}\left(P_{1} \times F_{1}\right)-\bar{F}_{1}\left(P_{1} \times P_{2}\right)-\bar{P}_{1}=0 \\
\mathrm{~B}^{\prime \prime} & 2 \bar{B}_{2}\left(P_{2} \times F_{1}\right)-R \bar{F}_{1}\left(P_{2} \times P_{1}\right)-\bar{P}_{2}=0 \\
\mathrm{C}^{\prime \prime} & 2 \bar{F}_{2}\left(F_{1} \times F_{1}\right)-R \bar{B}_{1}\left(F_{1} \times P_{1}\right) \\
& -R \bar{B}_{2}\left(F_{1} \times P_{2}\right)=0
\end{array}
$$

As the $\mathrm{D}$ scaling test is not influenced by the reciprocal differences it was applied without modification.

Two further tests involving the $L_{1}, L_{2}$ and $L_{3}$ generations have also been applied. One of them, $\bar{L}_{1}+\bar{L}_{2}-2 \bar{L}_{3}=0$, is a general test of epistasis and is valid for any population without any restrictions of gene or genotypic frequencies (Kearsey and Jinks, 1968; Bauman, 1959). This test is suitable for our purposes because the $F_{2}$ individuals have been used consistently as the maternal parent for each of the three generations. The other test involves the $L_{1}, L_{2}, F_{1}, R F_{1}, P_{1}$ and $P_{2}$ generations and tests the presence of epistasis as a significant departure of $\left\{\left(\bar{L}_{1}-\bar{L}_{2}\right)+\frac{1}{2}\left(\bar{F}_{1}-R \bar{F}_{1}\right)-\frac{1}{2}\left(\bar{P}_{1}-\bar{P}_{2}\right)\right\}$ from zero. These scaling tests have been labelled as $E$ and $F$. The results of these tests are given in table 5 .

These results show that, in general, non-allelic interactions are highly significant for mean performance and non-significant for environmental sensitivity. The only exceptions are the mean performance of character $T Y$ and the environmental sensitivities of $H 2, L N, F T, F H, I N L, L R$ and $H A F$. Thus, the inheritance of mean performance is more complex involving digenic and perhaps higher order interactions. The genetical control of environmental sensitivity, on the other hand, is simpler and may require only additive and dominance parameters for most of the traits.

\section{Linkage of the interacting genes}

The presence of significant non-allelic interactions for various combinations of character/mean performance allows us to test for linkage of interacting genes for all characters except $T Y$. The standard tests of Jinks (1978) however cannot be applied because they will be biased by the presence of reciprocal differences whenever the latter are significant. They have therefore been modified as follows to remove the biases:

$$
\begin{array}{cc}
\text { Test } & \text { Comparison } \\
1 & \frac{1}{2}\left(\bar{B}_{1}+R \bar{B}_{1}\right)-\bar{L}_{1}-\frac{1}{4}\left(\bar{F}_{1}-R \bar{F}_{1}\right) \\
2 & \frac{1}{2}\left(\bar{B}_{2}+R \bar{B}_{2}\right)-\bar{L}_{2}+\frac{1}{4}\left(\bar{F}_{1}-R \bar{F}_{1}\right) \\
3 & \frac{1}{2}\left(\bar{B}_{1}+R \bar{B}_{1}\right)+\frac{1}{2}\left(\bar{B}_{2}+R \bar{B}_{2}\right)-\bar{L}_{1}-\bar{L}_{2}
\end{array}
$$

The results of these tests and those of Jinks (1978) are given in table 6 for comparison. These tests have also been applied to those combinations of character/environmental sensitivity for which epistasis and reciprocal differences $(H 1, F T$ and $L N$ ) were observed to be significant. The results are also tabulated in the lower half of table 6.

Non-allelic interactions are also significant for the environmental sensitivities of $\mathrm{H} 2, \mathrm{FH}, I N L$, $L R$ and $H A F$. However, the standard tests of Jinks (1978) are still applicable to these combinations of character/environmental sensitivity because the reciprocal differences are non-significant in each case (table 3). As usual, the tests were conducted 
Table 5 Scaling tests and their significances for those combinations of character/mean performance and character/environmental sensitivity for which reciprocal differences were detected to be significant

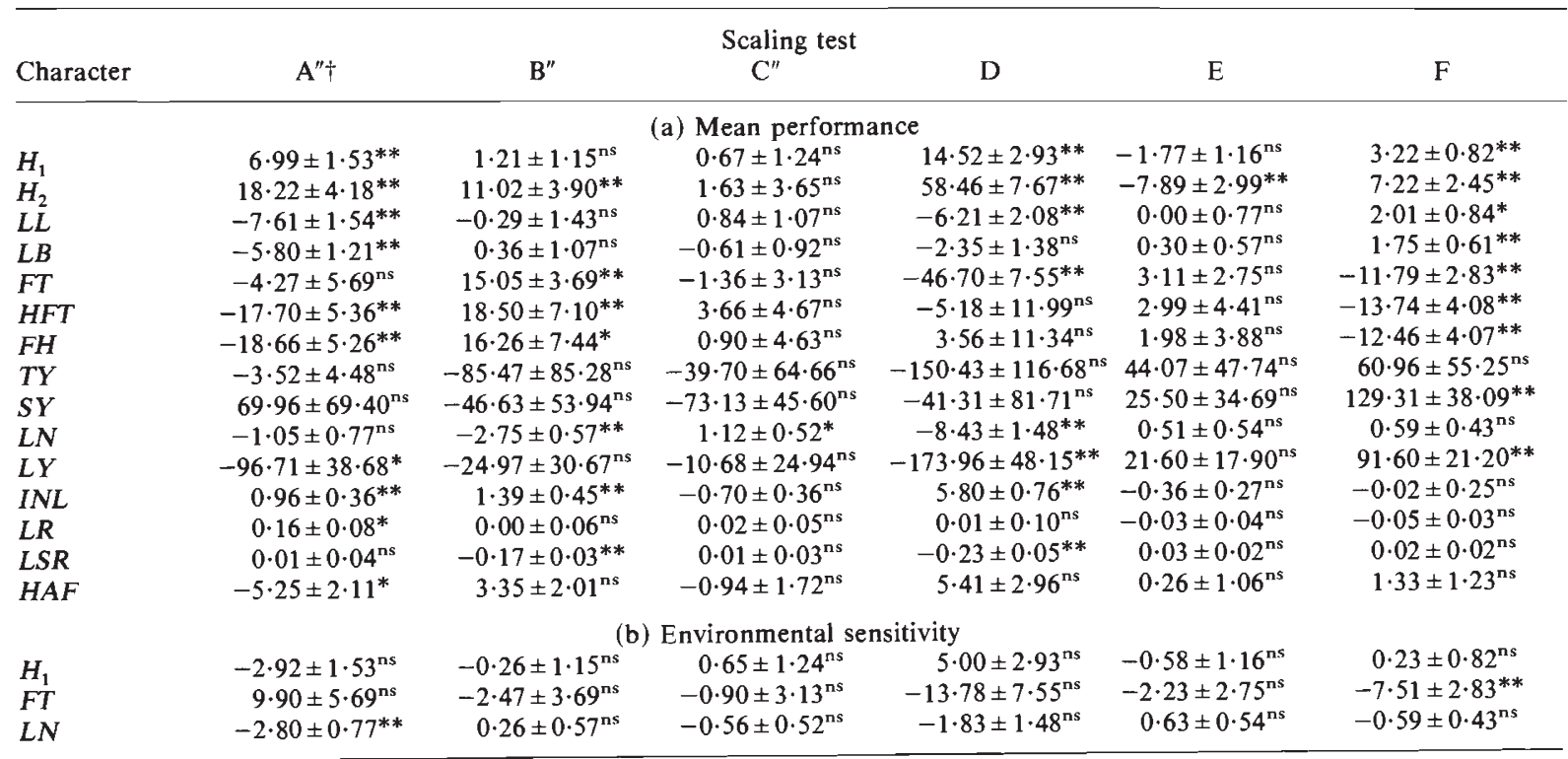

See table 3 for probability.

$\dagger$ See text for definitions of the tests.

Table 6 Tests for the detection of linkage between interacting genes for those combinations of characters/mean performance and character/environmental sensitivity for which both epistasis and reciprocal differences were detected to be significant

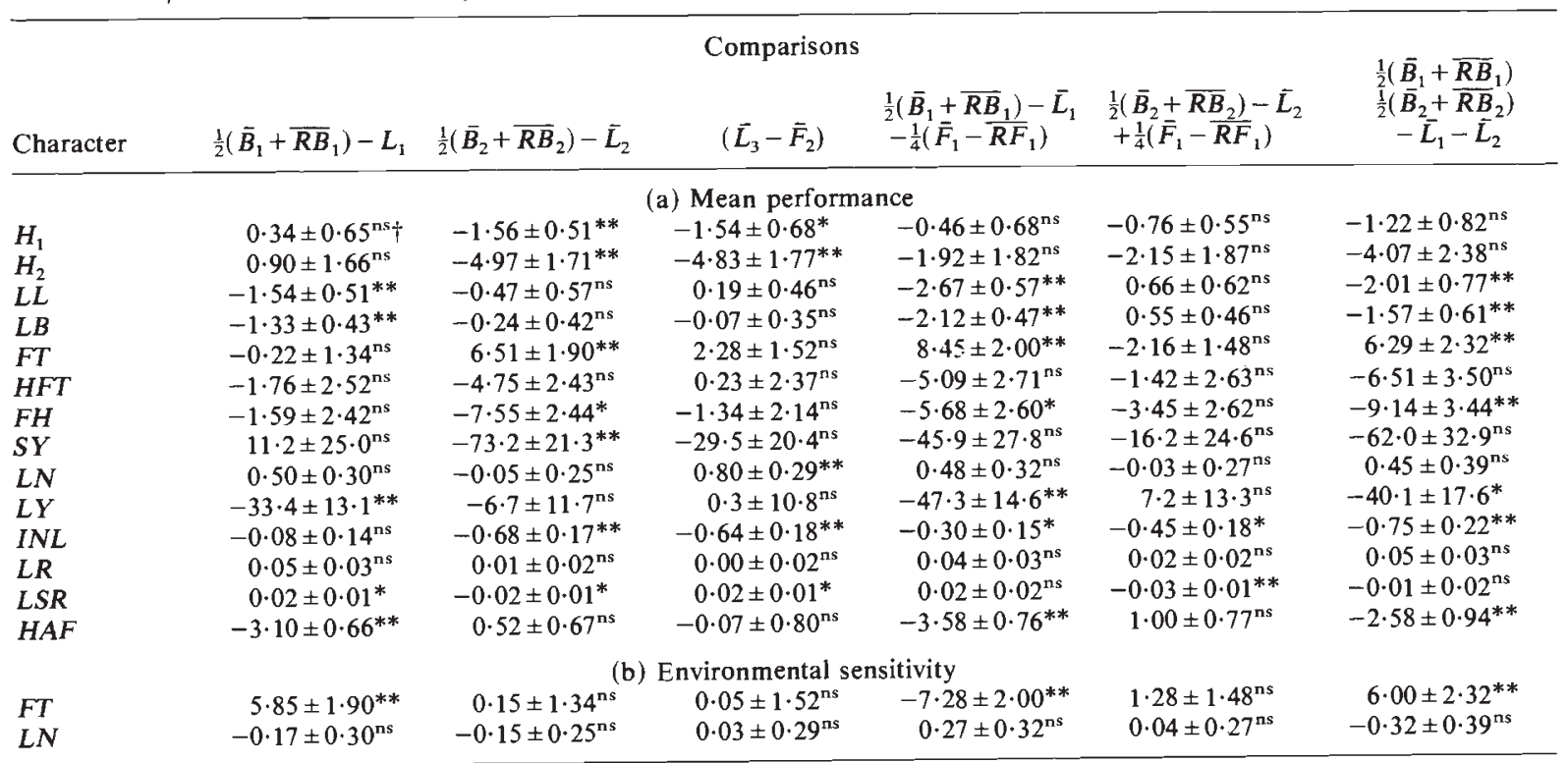

$\dagger$ See table 3 for probability. 
on the averages of the reciprocal crosses. None was significant hence there is no evidence of a linkage disequilibrium between the loci displaying epistasis.

\section{Components of first degree statistics}

The preliminary tests have shown that there is no significant epistasis for 8 of the 15 combinations of character/environmental sensitivity or for the mean performance of character $T Y$. The parameters of a simple additive genetic/dominance model and that of reciprocal effects should therefore fit these data. The models describing the expectations of the generation means in terms of $m,[d]$ and $[h]$ parameters have been given by Jinks and Perkins (1969). We shall utilise these models to obtain the weighted least squares estimates of the additive genetic and dominance components. Models describing the contributions of $[d]_{m}$ and $[h]_{m}$ to the family means of the reciprocal crosses are however available for the six basic generations only (Barnes, 1968; Mather and Jinks, 1971, 1982). We have therefore extended these models to the $F_{3}, L_{1}, L_{2}$ and $L_{3}$ generations so that they can be included in the estimation of the components of reciprocal effects. Since the $F_{2}$ is the mother of all of these generations it must be contributing $\frac{1}{2}[h]_{m}$ to their family means.

The scaling tests and modified tests of linkage also show that non-allelic interactions are significant and linkage is absent for the mean performances of $H 1, H 2, H F T, S Y, L N$ and $L R$ and the environmental sensitivities of $H 2, F H, I N L$, $L N, L R$ and $H A F$. Different combinations of additive $\times$ additive, additive $\times$ dominance and dominance $\times$ dominance interaction components $([i],[j]$ and $[l]$, respectively) will also be required in addition to $m,[d],[h],[d]_{m}$ and $[h]_{m}$ etc. to explain the variability in these data.

The appropriate models were fitted to these data and the estimates of the parameters together with tests of the adequacy of the models are tabulated in table 7. The models are adequate in all cases except for the mean performances of $\mathrm{H} 2$, $L N, T Y$ and $S Y$. The failures in these cases of what appeared from the scaling tests to be appropriate models could be due to:

(a) Failure of the scaling tests to detect non-allelic interactions (e.g. $T Y$ ) or linkage of interacting genes (e.g. $S Y$ ).

(b) Inadequacy of the model for reciprocal differences (Mather and Jinks, 1971, 1982). These possibilities we examine at the end of this section.
For the rest of the combinations of character/mean performance and character/environmental sensitivity the scaling tests have shown that models allowing for the linkage of the interacting genes are required. These models have been given by Jinks and Perkins (1969) and 11 parameters are needed to define the genetical expectations of the 10 generations included in the present study. If, however, we exclude $F_{3}$ the number of parameters is reduced to nine as $\left[p^{4} l\right]$ is not required and [pi] and $[\mathrm{pl}]$ are confounded. Since the number of parameters now equals the number of generations available, all can be estimated from a perfect fit solution. Further, the individual coefficients in the $J$ matrix (of $9 \times 9$ dimension) remain the same as those given by Jinks and Perkins (1969) except that $[\mathrm{pi}]$ and $[\mathrm{pl}]$ are replaced by one parameter, $[p i]+[p l]$.

This model was fitted simultaneously with $[d]_{m}$ and $[h]_{m}$ and found to be adequate for 5 out of 9 combinations. It failed for the mean performances of $L L, L B, F T$ and $L Y$. This failure however can only be attributed to the inadequacy of the simple additive/dominance model for the reciprocal effects because in each case nine genetical parameters were estimated from nine generations $\left(\chi_{(1)}^{2}\right.$ equals $9 \cdot 8,12 \cdot 6,8 \cdot 7$ and $16 \cdot 4$, respectively). Thus the model for reciprocal effects was extended to include the epistatic effects $\left([i]_{m}\right.$ and $\left.[l]_{m}\right)$ and interactions between the additive/dominance components of reciprocal differences and the progeny genotypes $\left(d . d_{m}, d . h_{m}, h . d_{m}\right.$ and $\left.h . h_{m}\right)$ following Mather and Jinks $(1971,1982)$. The results of fitting this extended model show that interactions of the reciprocal effects with the progeny genotype are indeed significant. These results are tabulated in table 8 .

The extended model was also applied to the mean performance scores of various generations for $H 2, L N, T Y$ and $S Y$ and the results are given in table 9. Clearly the interaction of the reciprocal effects with the progeny genotype rather than the linkage of the interacting genes was responsible for the failure of the $m,[d],[h],[i],[j],[l],[d]_{m}$ and $[h]_{m}$ model in these data.

\section{DISCUSSION AND CONCLUSION}

The main conclusion that we can draw from table 3 is that the parental varieties and their $F_{1}$ differ in their maternal and paternal contributions to their progeny. These differences, however, are largely confined to mean performance with little evidence of an effect on the environmental 


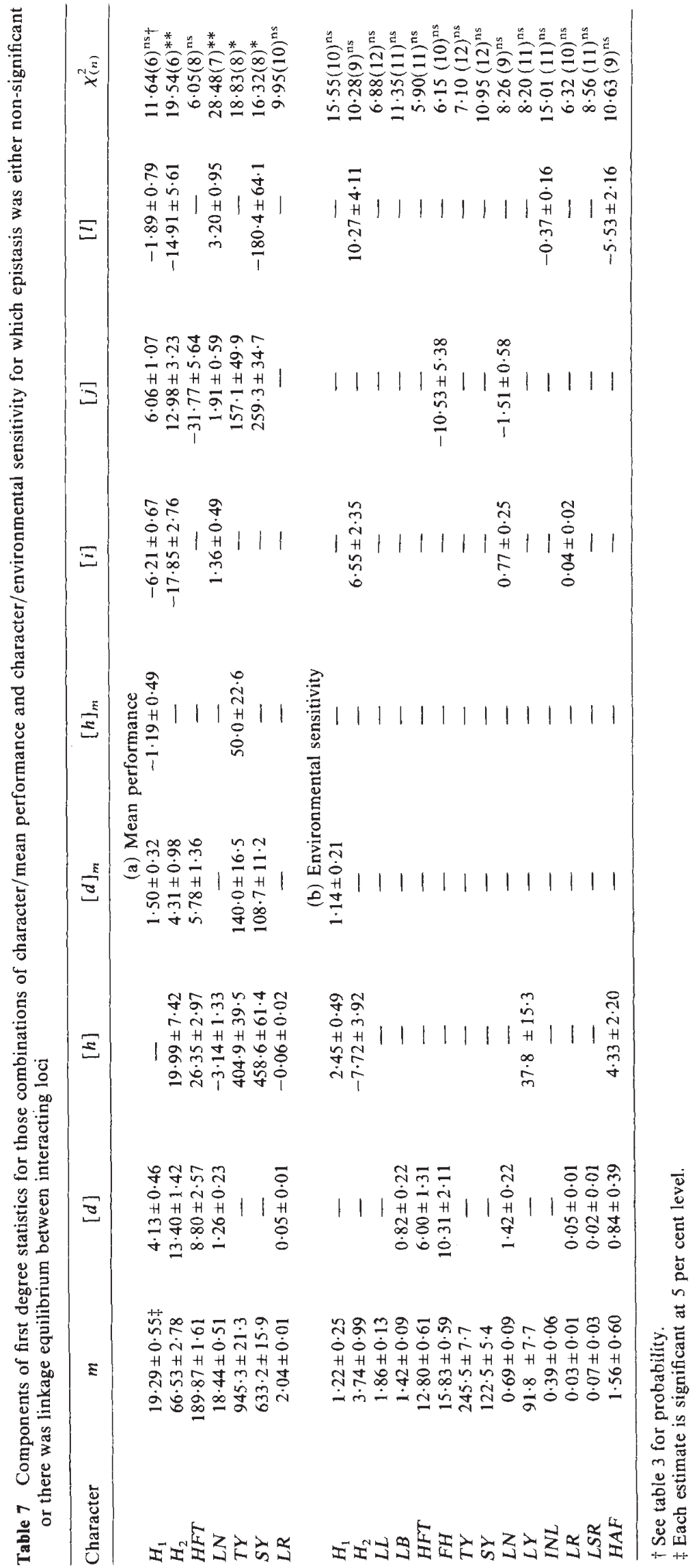




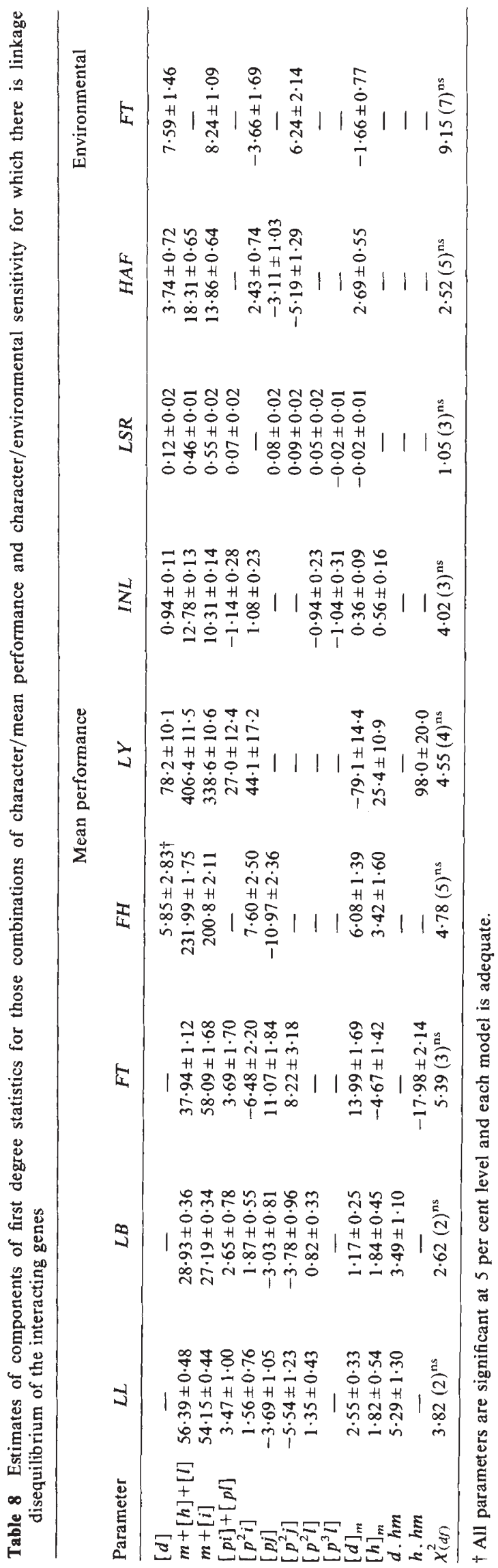


sensitivity. Furthermore, reciprocal differences attributable to the parental varieties, SCR and S3, are more often significant than those attributable to the reciprocal $F_{1}$ 's (14 and 8 combinations, respectively; see tables 7,8 and 9 and results). Using SCR rather than S3 as the maternal parent produces earliness $(F T)$, increases growth rate ( $H 1$ and $H 2$ ), leaf size ( $L L$ and $L B$ ), final height $(F H)$, total yield $(T Y)$, leaf yield $(L Y)$ and stem yield $(S Y)$, and reduces leaf stem ratio $(L S R)$.

Non-allelic interactions are significant for 14 combinations of character/mean performance and 7 combinations of character/environmental sensitivity (tables 4 and 5). Similarly, linkage of the interacting genes is significant more frequently for mean performance (for 11 characters) than for environmental sensitivity ( $F T$ only, table 6). Furthermore, the inclusion of $L_{1}, L_{2}$ and $L_{3}$ generations in the $\mathrm{A}, \mathrm{B}$ and $\mathrm{C}$ scaling tests has led to a reduction in the magnitudes of the standard errors and therefore increased the efficiency of the tests. Use of reciprocal crosses instead of their averages for detecting the non-allelic interactions in the presence of reciprocal differences has not, however, affected the combined efficiency of the scaling tests in these data (table 5).

The effects of reciprocal differences on the original $\frac{1}{2}\left(\bar{B}_{1}+R \bar{B}_{1}\right)-\bar{L}_{1}$ and $\frac{1}{2}\left(\bar{B}_{2}+R \bar{B}_{2}\right)-\bar{L}_{2}$ tests for linkage of interacting genes are shown in table 6. Out of 32 comparisons the original and modified tests differ for 9 . For six of these nine comparisons the original test is detecting the presence of linked interacting genes whose presence is not confirmed by the modified test. Hence for these six, reciprocal differences are presumably being misinterpreted as linkage of interacting genes. For the remaining three of the nine comparisons only the modified test detects their significance. Similar comparisons are not possible for the $\bar{F}_{2}-\bar{L}_{3}$ test because there is no simple method of correcting the biases incurred by this comparison. However, if we adjust its value by $\frac{1}{2}[h]_{m}$ (or $\frac{1}{2}[h]_{m}+\frac{1}{4} h . h_{m}$ as the case may be) we find that it also provides biased results on 5 out 16 occasions. Overall, the biases of these tests could have led us to draw wrong conclusions about the contributions of linked epistatic genes on at least three occasions (mean performances of $H 1, H 2$ and $S Y$ : see tables 6 and 8 ) had we not included the reciprocal crosses of the $F_{1}, B_{1}$ and $B_{2}$ generations in the experiment and followed the stringent procedures laid down by Mather and Jinks $(1971,1982)$ of testing the reciprocal differences before applying any other tests.

A general conclusion that we can draw from the present investigation is that the genetical control of mean performance is complex (tables 7,8 and 9). Of the 15 characters investigated, nonallelic interactions, linkage of the interacting genes and reciprocal differences are significant for 14,8 and 15 traits, respectively. Tables 7,8 and 9 also show that component $[d]$ is non-significant for five characters and its magnitude is relatively small for the remaining traits. This suggests that the alleles of like effect are largely dispersed in the parental varieties although they were selected as contrasting phenotypes. A large proportion of this phenotypic deviation is, however, attributable to $[d]_{m}$ (and $d{ }_{m}$ for $H 2$ and $S Y$ ) which is highly significant for 13 characters. Further, there is net directional

Table 9 Components of first degree statistics for those combinations of character/mean performance for which there was linkage equilibrium of the interacting genes but the appropriate model failed due to the inadequacy of the additive/dominance model for reciprocal differences

\begin{tabular}{|c|c|c|c|c|}
\hline Parameter & $\mathrm{H}_{2}$ & $\begin{array}{l}\text { Character } \\
L N\end{array}$ & $T Y$ & $S Y$ \\
\hline$m$ & $78 \cdot 56 \dagger \pm 1 \cdot 84$ & $20.05 \pm 0.22$ & $937 \cdot 4 \pm 20 \cdot 7$ & $606 \cdot 7 \pm 31 \cdot 6$ \\
\hline [d] & $13 \cdot 58 \pm 1 \cdot 42$ & $1 \cdot 55 \pm 0 \cdot 19$ & - & - \\
\hline$[h]$ & - & $-5.99 \pm 0.95$ & $367 \cdot 7 \pm 40 \cdot 7$ & $506 \cdot 1 \pm 96 \cdot 3$ \\
\hline$[i]$ & $-15 \cdot 58 \pm 3 \cdot 01$ & - & - & $120 \cdot 9 \pm 41 \cdot 2$ \\
\hline$[j]$ & $12 \cdot 55 \pm 3 \cdot 24$ & - & - & $263 \cdot 7 \pm 34 \cdot 8$ \\
\hline$[l]$ & $-6 \cdot 01 \pm 2 \cdot 61$ & $4 \cdot 39 \pm 0 \cdot 91$ & - & $-206 \cdot 0 \pm 81 \cdot 2$ \\
\hline$[d] m$ & $4.17 \pm 0.98$ & - & $155 \cdot 1 \pm 15 \cdot 5$ & $106 \cdot 0 \pm 11 \cdot 3$ \\
\hline$[h] m$ & $-6 \cdot 86 \pm 2 \cdot 02$ & $-7.07 \pm 1.45$ & - & - \\
\hline$d . d m$ & $-14 \cdot 07 \pm 3 \cdot 41$ & - & - & $-89 \cdot 0 \pm 36 \cdot 1$ \\
\hline d. hm & - & $1.07 \pm 0.39$ & - & - \\
\hline h. hm & - & $10 \cdot 88 \pm 2 \cdot 98$ & $560 \cdot 8 \pm 133 \cdot 2$ & - \\
\hline$[l] m$ & - & $1.51 \pm 0.65$ & $-225 \cdot 3 \pm 63 \cdot 8$ & - \\
\hline$\chi_{(n)}^{2}$ & $9 \cdot 15(5) \mathrm{ns}$ & $10 \cdot 63(5) \mathrm{ns}$ & $14 \cdot 18(8) \mathrm{ns}$ & $7 \cdot 23(6) \mathrm{ns}$ \\
\hline
\end{tabular}

$\uparrow$ All parameters are significant at 5 per cent level and each model is adequate. 
dominance which is negative for $F T, L N$ and $L S R$ and positive for the remaining traits. The relative signs (positive or negative) of $[h]$ and $[l]$ for those traits for which both components are significant and their inferred signs from the $m+[h]+[l]$, $m+[i],\left[p^{2} l\right]$ and $\left[p^{3} l\right]$ components when linkage is significant suggest that epistasis is predominantly of the duplicate type. Dominant dispersed alleles showing duplicate epistasis therefore must be responsible for the significant $F_{1}$ heterosis that is present for $H 2, F T, H F T, F H, S Y$ and $L Y$.

Genetical control of environmental sensitivity, in contrast, is comparatively simple (table 7). No detectable variation is present for leaf length $(L L)$, total yield $(T Y)$ and stem yield $(S Y)$ and the components $[d],[h],[i],[j]$ and $[l]$ are significant for eight, five, four, three and three characters, respectively. Flowering time $(F T)$ is the only character for which linkage of the interacting genes is significant. There is no significant heterosis except for flowering time $(F T)$ and leaf yield $(L Y)$ where the cause appears to be net directional dominance.

Finally, the estimates presented in tables 7 and 8 are those that satisfied all the criteria of an adequate model including the significances of reciprocal differences, non-allelic interaction and linked digenic interactions. It goes without saying that some alternative models were also statistically adequate but they did not satisfy the conditions imposed by the tests whose results are presented in tables 3 to 6 . This illustrates once more that simple statistical tests are in general more sensitive and more specific than sequential model fitting procedures specially when complex models are required (see Perkins and Jinks, 1970; Jinks, 1978; Eaves, Last, Martin and Jinks, 1977).
Acknowledgements This investigation was conducted in collaboration with Carrearas Rothmans Limited. D.T.C. and P.S.V. were supported by the SERC CASE Award and Leverhulme Postdoctoral Fellowship respectively.

\section{REFERENCES}

BARNES, B. W. 1968. Maternal control of heterosis for yield in Drosophila melanogaster. Heredity, 23, 563-572.

BRUMPTON, R. J., BOUGHEY, H. AND JINKS, J. L. 1977. Joint selection for both extremes of mean performance and of sensitivity to a macro-environmental variable. I. Family Selection. Heredity, 38, 219-226.

BAUMAN, L. F. 1959. Evidence of nonallelic interaction in determining yield, ear height and kernal row number in corn. Agron. J., 51, 531-534.

COOMBS, D. T. 1980. Biometrical genetics of tobacco. Ph. D. Thesis, University of Birmingham.

EAVES, L. J., LAST, K. A., MARTIN, N. G. AND JINKS, J. L. 1977. A progressive approach to non-additivity and genotypeenvironmental covariance in the analysis of human differences. Br. J. Math. Stat. Psychol., 30, 1-42.

JINKS, J. L. 1978. Unambiguous test for linkage of genes displaying nonallelic interactions for a metrical trait. Heredity, 40, 171-173.

JINKS, J. L. AND PERKINS, J. M. 1969. The detection of linked epistatic genes for a metrical trait. Heredity, 24, 465-475.

JINKS, J. L., JAYASEKARA, N. E. M. AND BOUGHEY, H. 1977. Joint selection for both extremes of mean performance and of sensitivity to a macroenvironmental variable. II Single Seed Descent. Heredity, 39, 345-355.

KEARSEY, M. J. AND JINKS, J. L. 1968. A general method for detecting additive, dominance and epistatic variation for metrical traits. I. Theory. Heredity, 23, 403-410.

MATHER, K. 1949. Biometrical Genetics, Ist edition, Methuen, London.

MATHER, K. AND JINKS, J. L. 1971. Biometrical Genetics, 2nd edition, Chapman and Hall, London.

MATHER, K. AND JINKS, J. L. 1982. Biometrical Genetics, 3rd edition, Chapman and Hall, London.

PERKINS, J. M. AND JINKS, J. L. 1970. Detection and estimation of genotype-environmental, linkage and epistatic components of variation for a metrical trait. Heredity, 25, 157177. 